\title{
Embodiment of Honeybee's Thermotaxis in a Mobile Robot Swarm
}

\author{
Daniela Kengyel ${ }^{1}$, Thomas Schmickl ${ }^{2}$, Heiko Hamann ${ }^{2}$, Ronald Thenius ${ }^{2}$, and \\ Karl Crailsheim ${ }^{2}$ \\ 1 University of Applied Sciences St. Poelten, Computersimulation, Austria \\ 2 Artificial Life Laboratory of the Department of Zoology, Karl-Franzens University \\ Graz, Universitätsplatz 2, A-8010 Graz, Austria, \\ daniela.kengyel@gmx.at, \{heiko.hamann, thomas.schmickl\}@uni-graz.at, \\ \{ronald.thenius, karl.crailsheim\}@uni-graz.at
}

\begin{abstract}
Searching an area of interest based on environmental cues is a challenging benchmark task for an autonomous robot. It gets even harder to achieve if the goal is to aggregate a whole swarm of robots at such a target site after exhaustive exploration of the whole environment. When searching gas leakages or heat sources, swarm robotic approaches have been evaluated in recent years, which were, in part, inspired by biologically motivated control algorithms. Here we present a bio-inspired control program for swarm robots, which collectively explore the environment for a heat source to aggregate. Behaviours of young honeybees were embodied on a robot by adding thermosensors in 'virtual antennae'. This enables the robot to perform thermotaxis, which was evaluated in a comparative study of an egoistic versus a collective swarm approach.
\end{abstract}

\section{Introduction}

\subsection{Motivation and Problem Formulation}

Eusocial insects are fascinating and inspiring group consisting of termites, bees, and ants, which live in well organized colonies. Colonies of honeybees are precisely regulated. For example the temperature in the brood nest is kept constant at approx. $36^{\circ} \mathrm{C}$. This is very important for the brood because otherwise this could lead to disorders in the development or even the loss of brood [1]. One challenge of a colony of honeybees in such a hive is, that the bees have to organize and orient themselves in a temperature gradient without the help of light. Such a temperature gradient is inhomogeneous and dynamic. This is a complicated challenge, because the antennae of honeybees are close to each other, but it is solved collectively by the bees [2]. To find a point of a special property, such as locating a field of heat or a leakage in a gas pipe, is a common challenge in robotics, especially if the environment is changing over time and no global gradient points to the target spot. One approach to tackle such challenges is using a bio-inspired approach, e.g. using a swarm to perform a parallelized and coordinated search. Following this idea, swarm robotics [3] emerged from combining autonomous robots with techniques originating from the field of swarm 
intelligence [4]. In swarm intelligent systems, the collective of agents performs decision making without a central unit that decides. As is summarized by [5], swarm intelligent solutions are robust, flexible, scalable and (computationally) cheap to implement on the individual. Camazine et. al. [6] showed that these properties apply also to biological swarms and social insect colonies, which exploit self-organizing processes to achieve swarm intelligence.

As an example, natural selection sharpened individual behaviour of bees in a way that colony fitness is maximized. Self-organisation and swarm intelligence can enhance this colony fitness significantly: In the following, we focus on groups of young honeybees which are an example of such a swarm-intelligent system. Young honeybees need a certain environmental temperature. The temperature in the hive varies usually between $32^{\circ} \mathrm{C}$ and $36^{\circ} \mathrm{C}$ while the optimal temperature for young honeybees is approximately $35^{\circ} \mathrm{C}$ [7]. This temperature is found at the brood nest of the hive, which is, preferred by the young honeybees [8]. Former experiments also showed that a single bee is mostly not able to detect the area of optimal temperature when the temperature gradient is rather flat [9].

\subsection{Derivation of the bio-inspired solution}

A model was reported in [10], that reproduces this behaviour of young bees without assuming any explicit communication between the bees. Inspired by the observed behaviour of young honeybees and based on this model, an algorithm for a swarm of robots was developed, which enables the robots to find an area with a special property collectively $[2,10]$. This typical (swarm) robotic task was frequently realized based on areas marked by light and using light sensors because this experiment configuration is supposed to be the technically simplest. We adopted the algorithm reported in [2] to consider the specific aspects of a temperature field in contrast to a light gradient field. The algorithm is based on robot-to-robot approaches. For simplicity we call it the BEECLUST algorithm further on. By using heat instead of light the robots' environment gets closer to the original bio-inspired situation young honeybees are faced with. Due to the fact that heat differs in its physical characteristics compared to light (e.g., warming up period, thermal diffusion, turbulences in the airflow) we show here how physical properties of stimuli are reflected in the swarm's control algorithm.

The typical engineering approach is very different to this swarm-intelligent approach: In his book Braitenberg describes algorithms, such as a simple gradient ascent, of robots which are sensitive to the environment [11]. There are several works describing complex approaches with cooperating sensor networks and robot groups (e.g., [12]). The sensor network measures the temperature gradient and communicates within the robot swarm. The robots are able to localize

themselves globally and perform a simple gradient ascent. A single robot that produces heat trails and follows them is reported in [13]. 


\section{Material \& Method}

\subsection{Hardware \& Electronics}

For our robot experiments, we needed a small robot with two temperature sensors. A good solution was to upgrade an out-of-the-shelf robot with an according sensor system. Additional to an extensible I/O-board, the Hemisson robot has six infrared-sensors, which we used for collision avoidance and for the identification of other robots. Three of these sensors point to the front, one to each side and one to the back. The locomotion is realized by two wheels with a differential drive system. In addition, we developed a circuit processing the measured temperature. The circuit with temperature sensors is able to detect temperatures in a range of $140^{\circ} \mathrm{C}$. We re-mapped this range to the relevant temperature interval $\left[10^{\circ} \mathrm{C}, 60^{\circ} \mathrm{C}\right]$ of honeybees, represented by digital sensor values on the interval $e \in[50,225]$. Our measurements indicate that the sensor's voltage is approx. linear within this temperature interval. Using an instrumentation amplifier the measured signal was amplified for processing the data in an easier way. The advantage of an instrumentation amplifier compared to the operational amplifier is that the former is regulated by a single resistor without affecting the circuit. Fig. 1A shows the Hemisson with the temperature sensors.

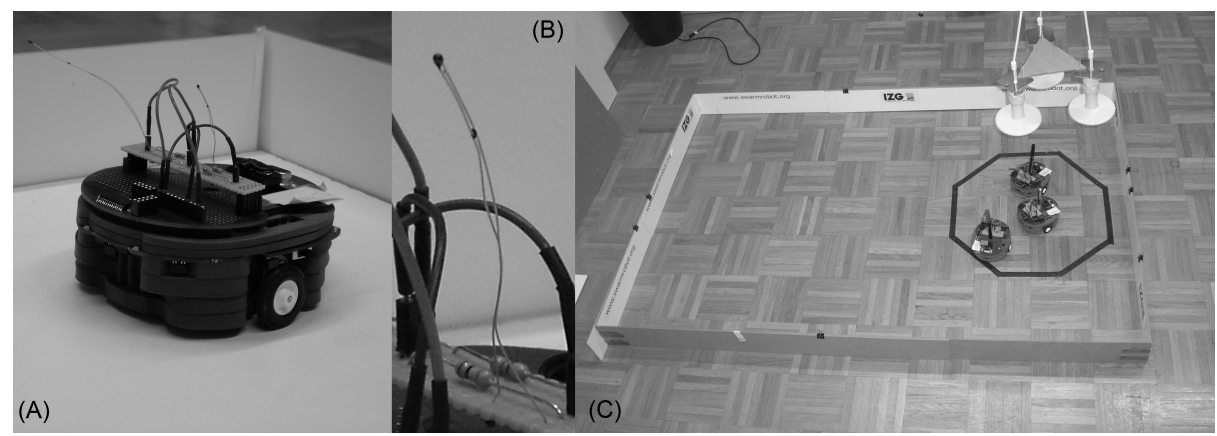

Fig. 1. A: Robot "Hemisson" with temperature sensors. B: Temperature sensor of the Hemisson. C: Robots underneath the heat lamps in the arena.

\subsection{Algorithms}

Naturally, there are many possibilities to implement the aggregation of autonomous agents at a certain target area. Here, we want to analyze the properties of the BEECLUST algorithm when working with fields of temperature instead of light gradients. This bio-inspired algorithm is different from anything that could be considered to be a standard approach. Actually, it might even be perceived as counter-intuitive. However, we hypothesize that it is robust and efficient in multirobot scenarios. In order to test this hypothesis we compare the BEECLUST to a rather classic approach of a simple gradient ascent algorithm (GAA). 
BEECLUST Algorithm: BEECLUST is adopted from young honeybees' navigation behaviour. In principle there are two strategies how young honeybees find the optimal temperature: In a rather steep gradient field, the individual behaviour of each single bee is effective and dominates. In a rather flat gradient, a single bee is mostly not able to find the spot of optimal temperature. The following group behaviour of young honeybees in such an environment was observed [2]: The bees walk around randomly until they meet another bee. Then they stop and wait. After a certain time the bees walk around randomly again. This process forms clusters of bees. The higher the temperature is, the longer the bees wait. Hence, the cluster sizes and numbers increase over time in the warmer area whereas they decrease in the colder area. To realize such a behaviour the robots have to perform several different tasks: random movement, discrimination between the wall and other robots, avoidance of collisions, measurement of local temperature and calculation of the waiting time.

Our program on the Hemisson robot mimics the minimal individual behaviour extracted from honeybees: A robot drives forward until it detects an object in the front. If this object is an obstacle (e.g., a wall) the robot turns in a random direction by a random angle. This turning angle is uniformly randomly distributed between 40 degrees and 140 degrees in both directions. If the detected object is another robot, the robot stops, measures the temperature, and calculates the time to wait. The robot-to-robot detection is realized by the help of passive sensing using infrared sensors. Passive sensing means, in contrast to active sensing, that no infrared light is emitted. If the sensor receives IR without emitting IR there is only one possibility: The IR-light was emitted by another robot. The dependency of the waiting-time on the measured temperature is also an important factor and is described with a sigmoid curve. The following equation is used to map the sensor values $e$ to waiting times (for details see [2]):

$$
w(e)=\frac{w_{\max } e^{2}}{e^{2}+\theta},
$$

where $\theta$ indicates the steepness of the resulting curve. See table 1 for characteristics of the robot and other parameters that were used in this work in comparison to parameters of the original BEECLUST [2].

Table 1. Parameters

\begin{tabular}{lcc}
\hline & temperature & light \\
\hline speed of the robot & $3 \mathrm{~cm} / \mathrm{s}$ & $30 \mathrm{~cm} / \mathrm{s}$ \\
range of infrared sensors & $4 \mathrm{~cm}$ & $6 \mathrm{~cm}$ \\
robot size & $12 \times 13 \mathrm{~cm}$ & $3 \times 3 \mathrm{~cm}$ \\
max. waiting time $w_{\max }$ & $180 \mathrm{~s}$ & $66 \mathrm{~s}$ \\
interval of the sensor value $e$ & {$[50,225]$} & {$[0,255]$} \\
waiting time function offset $\theta$ & 21,000 & 7,000 \\
\hline
\end{tabular}


GAA - Gradient-Ascent Algorithm: The GAA represents a simple straightforward approach to thermotaxis. The collision avoidance is implemented in the same manner as described above. The only difference is that there is no robotto-robot detection implemented and therefore no waiting-time is calculated. The GAA works as follows: The robot measures the temperature with a frequency of two measurements per second. If one sensor reports a difference of temperature greater than a threshold of 2 in the digital sensor value $e$ (to achieve a certain robustness against fluctuations), the robot changes its direction and starts to turn to the warmer area until the difference between the two sensors has vanished. A difference of 2 units in the digital sensor value corresponds to a difference in the temperature of about $1^{\circ} \mathrm{C}$.

\subsection{Setup of the experiment}

Experiment 1: To compare both algorithms we used three Hemisson robots and built a rectangular arena as shown in Fig. 1C. The arena had a size of $150 \mathrm{~cm} \times 120 \mathrm{~cm}$. Over the right side of the arena we placed three infrared heat lamps at a height of $44 \mathrm{~cm}$. Each of them had a power of 250 watt. The lamps generated $30^{\circ} \mathrm{C}$ in a height of $10 \mathrm{~cm}$ (where the robots measure the temperature) above the target area. The ambient air temperature in the arena (where no heat lamps were) was around $27^{\circ} \mathrm{C}$. The target zone underneath the heat lamps was marked with a surrounding black line as seen in Fig. 1C. It covered about an eighth of the arena. Initially, the robots (depending on the scenario: one, two, or three) are positioned at the side of the arena opposite to the target zone. In case of BEECLUST, many robot-to-robot detections occur in the first seconds. However, they do not remain there because the waiting-time in the cold part of the arena is very small (mostly no waiting-time).

It was important to ensure that the ambient air temperature did not significantly change during the experiment. Such changes were prevented by heating up the room to $27^{\circ} \mathrm{C}$ before our experiments started. To ensure the same initial conditions the temperature was measured before each experiment started. In these experiments, the time each robot spent inside of the target zone was measured. Each experiment lasted 14 minutes.

Experiment 2: In an additional experiment the probability of staying within the target area after a robot-to-robot approach was measured after a time of 25 seconds. In case of the BEECLUST, not every robot-to-robot detection results in a successful detection of the other robot due to noise and blind spots of the infrared sensors. In case of the GAA, two approaching robots interfere with each other and might "push" each other out of the target area when trying to avoid collisions. In this experiment we had five different setups: a) one GAA robot, $b$ ) two GAA robots, $c$ ) three GAA robots, $d$ ) two BEECLUST robots and $e$ ) three BEECLUST robots . The robots started vis-á-vis and where placed $10 \mathrm{~cm}$ outside of the target area. The third robot in experiment $c$ and $e$ was placed inside of the target area and was programmed to stay immobile. Therefore, it was not included in the determination of the results. 


\section{Results \& Discussion}

Fig. 2 shows the results of our three test runs in experiment 1 ( $N=6$ repetitions). Three robots with the GAA have spent $25.4 \%$ (mean) of time inside of the target zone. This time span is quite short compared to the mean of $49.7 \%$ in the runs with a single GAA robot. This is explained by the consequences of the competition for space underneath the heat lamps: If one robot is inside the target zone and a second robot tries to get into the target zone as well, it may happen that they approach each other. The collision avoidance behaviour might lead to a turn and the robot might leave the target area.

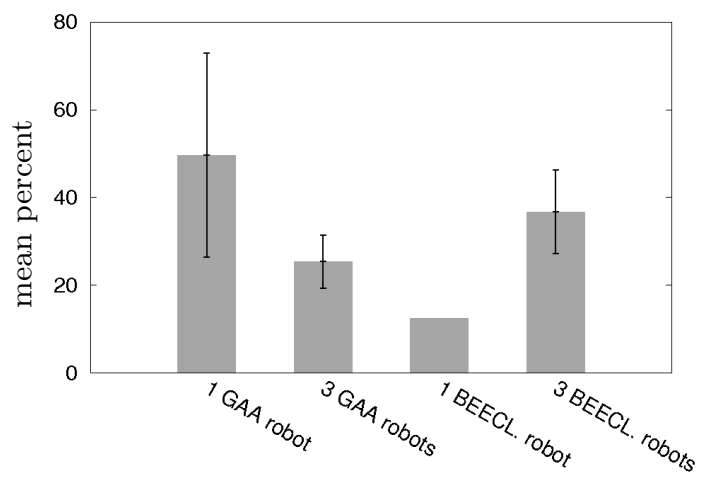

Fig. 2. Mean of the percentage of the total time a robot spent inside of the target zone (see Fig. 1C). Error bars show the standard deviation, $N=6$ per setting.

One single BEECLUST robot spent an eighth of the time in the target area, because the target area covers an eighth of the arena. This was calculated statistically because one single robot cannot stop underneath the heat lamps. Three BEECL. robots yielded a result with a mean of $36.7 \%$. In comparison to the GAA the swarm-intelligent BEECL. has a higher success rate. If there is no heat source in the arena the three robots do not aggregate for both algorithms.

In test runs with three robots, three cases of robot-to-robot encounters in the target area can occur, which are tested in experiment 2. Fig. 3 shows the relative frequency of a robot to stay in the target area. The accounted final state was measured 25 seconds after the start leaving enough time for a robot to get out of the target zone: One single GAA robot stayed inside of the target zone with a frequency of 0.75 per trial. Two GAA robots remained there with a frequency of 0.67 per trial whereas the frequency was 0.54 per trial in the experiment with three GAA robots. 2 BEECL. robots had a frequency of 0.91 per trial and 3 BEECL. robots remained in the target area with a frequency of 0.83 per trial. Significant differences were determined for the comparisons: 1 BEECL. and all other comparable settings (Chi-square test, $P<0.0001$ ), 2 GAA and 2 BEECL. $\left(P<0.0330, \chi^{2}=4.547\right)$, and 3 GAA and 3 BEECL. $\left(P<0.0293, \chi^{2}=4.752\right)$. 


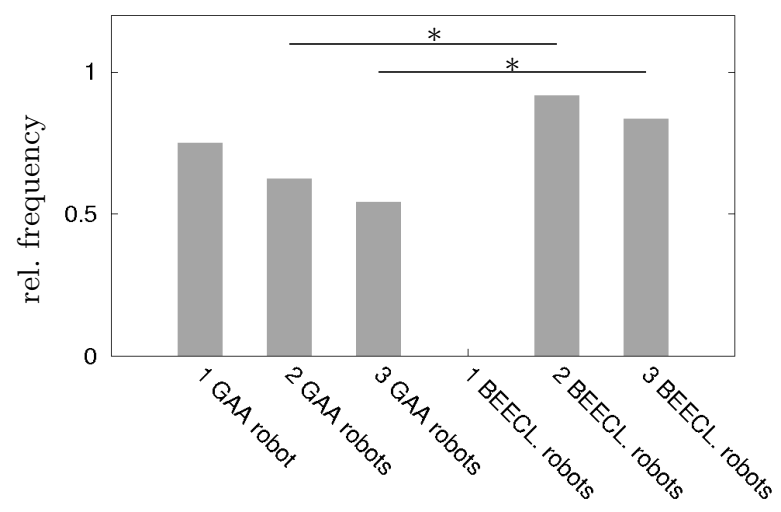

Fig. 3. Comparison of the relative frequency of robots that stay in the target zone for both algorithms and for all possible robot-to-robot encounters, $N=12$ per setting.

The probability of the GAA robots remaining in the target zone decreases with increasing number of robots. It is expected that with a higher number of robots the probability will decrease further because the limited resource "space" in the target area will get more scarce. Although this is true for the BEECLUST robots as well, they have a higher probability of staying in the target area.

\section{Conclusion and Outlook}

As expected the use of heat instead of light brings along significant challenges in designing an efficient robotic swarm due to different physical characteristics. Differences are observed, e.g., in the shape of the temperature gradient, in the time scale of diffusion and the time delays in the measurement of temperatures. This had to be reflected in the control algorithm by slower motion/turns and longer waiting periods. The time delay of measurement originates from the temperature sensors because they have to heat themselves up to decrease their resistance. If the robot is not exposed to heat long enough it measures a lower temperature and so the calculated waiting-time is shorter. As a consequence the aggregated robot swarm is not as robust as it could be in a light gradient.

A major challenge was the inhomogeneity concerning the spreading of heat and air flows. Due to the fact that hot air ascends, the heat lamps generate a lot of waste heat which heats up the ambient air instead of the desired area in the robot arena. Between experiment runs the initial conditions (defined temperatures at the target area, in the ambient air, etc.) needed to be re-established. Otherwise the air temperature saturates close to the temperature in the target zone and the robots cannot identify the target area anymore. This would influence the effectivity of the algorithms critically. Despite these characteristics, which increase the difficulty of this task, the robots were able to locate the target area beneath the heat lamps using the BEECLUST. This was possi- 
ble although the configuration of the infrared-sensors of the Hemisson, used for collision-detection and the detection of another robot, have blind spots.

In this paper we focused on building robots which are able to perform thermotaxis. The experiments were used as a proof of concept. For an exhaustive analysis are multiple robots, a larger arena and a longer period of time required. We will continue to use these robots as a model to emulate natural organisms such as honeybees. Furthermore we will test the robustness of bio-inspired algorithms to obstacles and disturbances of the gradient.

\section{Acknowledgements}

This work was supported by: EU-IST-FET project 'SYMBRION', no. 216342; EU-ICT project 'REPLICATOR', no. 216240. Austrian Science Fund (FWF) research grants: P15961-B06 and P19478-B16.

\section{References}

1. Stabentheiner, A., Schmaranzer, S., Heran, H., Ressl, R.: Verändertes thermopräferendum von jungbienen durch intoxikation mit roxion-s (dimethoat). Mitteilungen der Deutschen G. f. allg. u. angew.Entomologie 6 (1988) 514-520

2. Schmickl, T., Thenius, R., Möslinger, C., Radspieler, G., Kernbach, S., Crailsheim, K.: Get in touch: Cooperative decision making based on robot-to-robot collisions. Autonomous Agents and Multi-Agent Systems 18(1) (February 2008) 133-155

3. Şahin, E.: Swarm robotics: From sources of inspiration to domains of application. In Şahin, E., Spears, W.M., eds.: Swarm Robotics - SAB 2004 International Workshop. Volume 3342 of LNCS., Berlin, Germany, Springer-Verlag (2005) 10-20

4. Kennedy, J., Eberhart, R.C.: Swarm Intelligence. (Morgan Kaufmann Series in Evolutionary Computation). Morgan Kaufmann (2001)

5. Millonas, M.M.: Swarms, phase transitions, and collective intelligence. In Langton, C.G., ed.: Artificial Life III, Reading, MA, Addison-Wesley (1994)

6. Camazine, S., Deneubourg, J.L., Franks, N.R., Sneyd, J., Theraulaz, G., Bonabeau, E.: Self-Organizing Biological Systems. Princeton Univ. Press (2001)

7. Jones, J.C., Myerscough, M.R., Graham, S., Oldroyd, B.P.: Honey bee nest thermoregulation: Diversity promotes stability. Science 305 (2004) 402-404

8. Bujok, B.: Thermoregulation im Brutbereich der Honigbiene apis mellifera carnica. Dissertation, Universität Würzburg (2005)

9. Szopek, M., Radspieler, G., Schmickl, T., Thenius, R., Crailsheim, K.: Recording and tracking of locomotion and clustering behavior in young honeybees (apis mellifera). In Spink, et al., eds.: Proc. Measuring Behavior. Vol. 6. (2008) 327

10. Schmickl, T., Hamann, H.: BEECLUST: A swarm algorithm derived from honeybees. In Xiao, Y., Hu, F., eds.: Bio-inspired Computing and Communication Networks. Routledge (January 2010)

11. Braitenberg, V.: Vehicles: Experiments in synthetic psychology. MIT Press (1984)

12. Kantor, G., et al.: Distributed search and rescue with robot and sensor teams. In: Proc. 4th Int. C. on Field and Service Robotics. (July 2006) 529-538

13. Russell, R.: Heat trails as short-lived navigational markers for mobile robots. In: Proceedings of the IEEE International Conference on Robotics and Automation. Volume 4. (1997) 3534-3539 